OPEN $\curvearrowright$ ACCESS

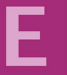
COMMUNICATIONS

ISSN 2056-9890

\section{Crystal structure of 4,6-dichloro-5- methylpyrimidine}

\author{
Meriem Medjani, ${ }^{a} *$ Noudjoud Hamdouni, ${ }^{a}$ Ouarda Brihi, \\ Ali Boudjada ${ }^{a}$ and Jean Meinnel ${ }^{b}$ \\ a Laboratory of Crystallography, Department of Physics, University Mentouri Brothers \\ Constantine, 25000 Constantine, Algeria, and ${ }^{\mathbf{b}}$ UMR 6226 CNRS University of \\ Rennes 1 'Chemical Sciences Rennes', 'Team Systems and Synthetic Condensed \\ Electroactive', 263 Avenue du General Leclerc, F-35042 Rennes, France
}

Received 11 December 2015; accepted 14 December 2015

Edited by H. Stoeckli-Evans, University of Neuchâtel, Switzerland

The title compound, $\mathrm{C}_{5} \mathrm{H}_{4} \mathrm{Cl}_{2} \mathrm{~N}_{2}$, is essentially planar with an r.m.s. deviation for all non-H atoms of $0.009 \AA$. The largest deviation from the mean plane is 0.016 (4) $\AA$ for an $\mathrm{N}$ atom. In the crystal, molecules are linked by pairs of $\mathrm{C}-\mathrm{H} \cdots \mathrm{N}$ hydrogen bonds, forming inversion dimers, enclosing an $R_{2}^{2}(6)$ ring motif.

Keywords: crystal structure; pyrimidine; inversion dimers; C- $\mathrm{H} \cdots \mathrm{N}$ hydrogen bonding.

CCDC reference: 1442378

\section{Related literature}

For the applications of pyrimidine derivatives as pesticides and pharmaceutical agents, see: Condon et al. (1993); as agrochemicals, see: Maeno et al. (1990); as antiviral agents, see: Gilchrist (1997); as herbicides, see: Selby et al. (2002); Zhu et al. (2007); and for applications of organoselenide compounds, see: Ip et al. (1997). For the crystal structure of 5-methylpyrimidine, see: Furberg et al. (1979).

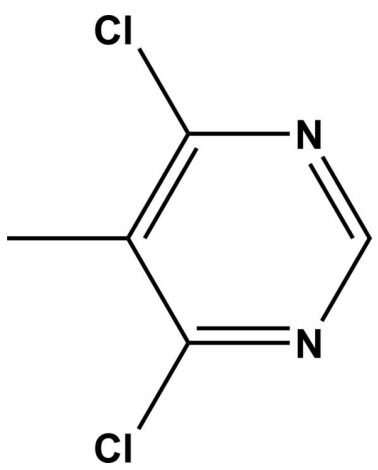

\section{Experimental}

2.1. Crystal data

$\mathrm{C}_{5} \mathrm{H}_{4} \mathrm{Cl}_{2} \mathrm{~N}_{2}$

$M_{r}=163.00$

Monoclinic, $P 2_{1} / n$

$a=7.463(5) \AA$

$b=7.827(5) \AA$

$c=11.790(5) \AA$

$\beta=93.233(5)^{\circ}$

$$
\begin{aligned}
& V=687.6(7) \AA^{3} \\
& Z=4 \\
& \text { Mo } K \alpha \text { radiation } \\
& \mu=0.85 \mathrm{~mm}^{-1} \\
& T=293 \mathrm{~K} \\
& 0.11 \times 0.10 \times 0.08 \mathrm{~mm}
\end{aligned}
$$

\subsection{Data collection}

Oxford Diffraction Xcalibur, Eos diffractometer

Absorption correction: multi-scan (CrysAlis PRO; Oxford Diffraction, 2013)

$T_{\min }=0.922, T_{\max }=0.934$

2347 measured reflections 1228 independent reflections 791 reflections with $I>2 \sigma(I)$ $R_{\text {int }}=0.099$

\subsection{Refinement}

$R\left[F^{2}>2 \sigma\left(F^{2}\right)\right]=0.068$

$w R\left(F^{2}\right)=0.173$

$S=1.01$

1228 reflections

83 parameters

$\mathrm{H}$-atom parameters constrained

$\Delta \rho_{\max }=0.39{\mathrm{e} \AA^{-3}}^{-3}$

$\Delta \rho_{\min }=-0.38$ e $\AA^{-3}$

Table 1

Hydrogen-bond geometry $\left(\AA{ }^{\circ}\right)$.

\begin{tabular}{lllll}
\hline$D-\mathrm{H} \cdots A$ & $D-\mathrm{H}$ & $\mathrm{H} \cdots A$ & $D \cdots A$ & $D-\mathrm{H} \cdots A$ \\
\hline $\mathrm{C} 2-\mathrm{H} 2 \cdots \mathrm{N}^{\mathrm{i}}$ & 0.93 & 2.66 & $3.468(6)$ & 146 \\
\hline
\end{tabular}

Symmetry code: (i) $-x,-y+1,-z+1$.

Data collection: CrysAlis PRO (Oxford Diffraction, 2013); cell refinement: CrysAlis PRO; data reduction: CrysAlis PRO; program(s) used to solve structure: SIR97 (Altomare et al., 1999); program(s) used to refine structure: SHELXL2014/6 (Sheldrick, 2015); molecular graphics: PLATON (Spek, 2009) and Mercury (Macrae et al., 2008); software used to prepare material for publication: SHELXL2014/6 and PLATON.

\section{Acknowledgements}

This work is supported by the Laboratoire de Cristallographie, Département de Physique, Université Mentouri-Constantine, Algeria, and the UMR 6226 CNRS-Université Rennes 1 'Sciences Chimiques de Rennes', France. We would also like to thank Mr F. Saidi, Engineer at the Université MentouriConstantine, for assistance with the data collection.

Supporting information for this paper is available from the IUCr electronic archives (Reference: SU5261).

\section{References}

Altomare, A., Burla, M. C., Camalli, M., Cascarano, G. L., Giacovazzo, C., Guagliardi, A., Moliterni, A. G. G., Polidori, G. \& Spagna, R. (1999). J. Appl. Cryst. 32, 115-119. 
Condon, M. E., Brady, T. E., Feist, D., Malefyt, T., Marc, P., Quakenbush, L. S., Rodaway, S. J., Shaner, D. L. \& Tecle, B. (1993). Brighton Crop Prot. Conf. Weeds, pp. 41-46 Alton, Hampshire, England: BCPC Publications.

Furberg, S., Grøgaard, J. \& Smedsrud, B. (1979). Acta Chem. Scand. 33b, 715724.

Gilchrist, T. L. (1997). Heterocycl. Chem. 3rd ed., pp. 261-276. Singapore: Addison Wesley Longman.

Ip, C., Lisk, D. J., Ganther, H. \& Thompson, H. J. (1997). Anticancer Res. 17, 3195-3199.

Macrae, C. F., Bruno, I. J., Chisholm, J. A., Edgington, P. R., McCabe, P., Pidcock, E., Rodriguez-Monge, L., Taylor, R., van de Streek, J. \& Wood, P. A. (2008). J. Appl. Cryst. 41, 466-470.
Maeno, S., Miura, I., Masuda, K. \& Nagata, T. (1990). Brighton Crop Protection Conference on Pests and Diseases, pp. 415-422 Alton, Hampshire, England: BCPC Publications.

Oxford Diffraction (2013). CrysAlis PRO. Oxford Diffraction Ltd., Abingdon, UK.

Selby, T. P., Drumm, J. E., Coats, R. A., Coppo, F. T., Gee, S. K., Hay, J. V., Pasteris, R. J. \& Stevenson, T. M. (2002). ACS Symposium Series, Vol. 800, Synthesis and Chemistry of Agrochemicals VI, pp. 74-84. Washington DC: American Chemical Society.

Sheldrick, G. M. (2015). Acta Cryst. C71, 3-8.

Spek, A. L. (2009). Acta Cryst. D65, 148-155.

Zhu, Y.-Q., Zou, X.-M., Li, G.-C., Yao, C.-S. \& Yang, H.-Z. (2007). Chin. J. Org. Chem. 27, 753-757. 


\section{supporting information}

Acta Cryst. (2015). E71, o1073-o1074 [https://doi.org/10.1107/S2056989015024020]

Crystal structure of 4,6-dichloro-5-methylpyrimidine

Meriem Medjani, Noudjoud Hamdouni, Ouarda Brihi, Ali Boudjada and Jean Meinnel

\section{S1. Comments}

Pyrimidines have interesting biological properties with applications as pesticides, pharmaceutical agents (Condon et al., 1993; Maeno et al., 1990) and are also interesting from a biochemical pint of view and applications of organoselenide compounds (Ip et al., 1997). Pyrimidine derivatives have been developed as antiviral agents, such as AZT, which is the anti-AIDS drug most widely used (Gilchrist, 1997). Recently, a new series of highly substituted pyrimidine herbicides have been reported (Selby et al., 2002; Zhu et al., 2007). In the present study, we were interested in examining a derivative of pyrimidine with a methyl substituent surrounded by two chlorine atoms.

The molecular structure of the title compound is shown in Fig. 1. The molecule is planar, as is typical in benzenes substituted by halogen atoms and methyl groups, with an r.m.s. deviation for all non-H atoms of $0.009 \AA$. The largest deviation from the mean plane is 0.016 (4) $\AA$ for atom N3. The bond distances and bond angles in the molecule are similar to those reported for 5-methylpyrimidine (Furberg et al., 1979).

In the crystal, molecules are linked by a pair of $\mathrm{C}-\mathrm{H} \cdots \mathrm{N}$ hydrogen bonds forming inversion dimers (Table 1 and Fig. $2)$, enclosing an $\mathrm{R}_{2}^{2}(6)$ ring motif.

\section{S2. Synthesis and crystallization}

The commercially available title compound (Sigma-Aldrich) was recrystallized from ethanol giving colourless prismatic crystals.

\section{S3. Refinement}

Crystal data, data collection and structure refinement details are summarized in Table 2. All $\mathrm{H}$ atoms were localized in a difference Fourier map but introduced in calculated positions and treated as riding: $\mathrm{C}-\mathrm{H}=0.93-0.96 \AA$ with $U_{\text {iso }}(\mathrm{H})=$ $1.5 U_{\mathrm{eq}}(\mathrm{C}-\mathrm{methyl})$ and $1.2 U_{\mathrm{eq}}(\mathrm{C})$ for other $\mathrm{H}$ atoms. 


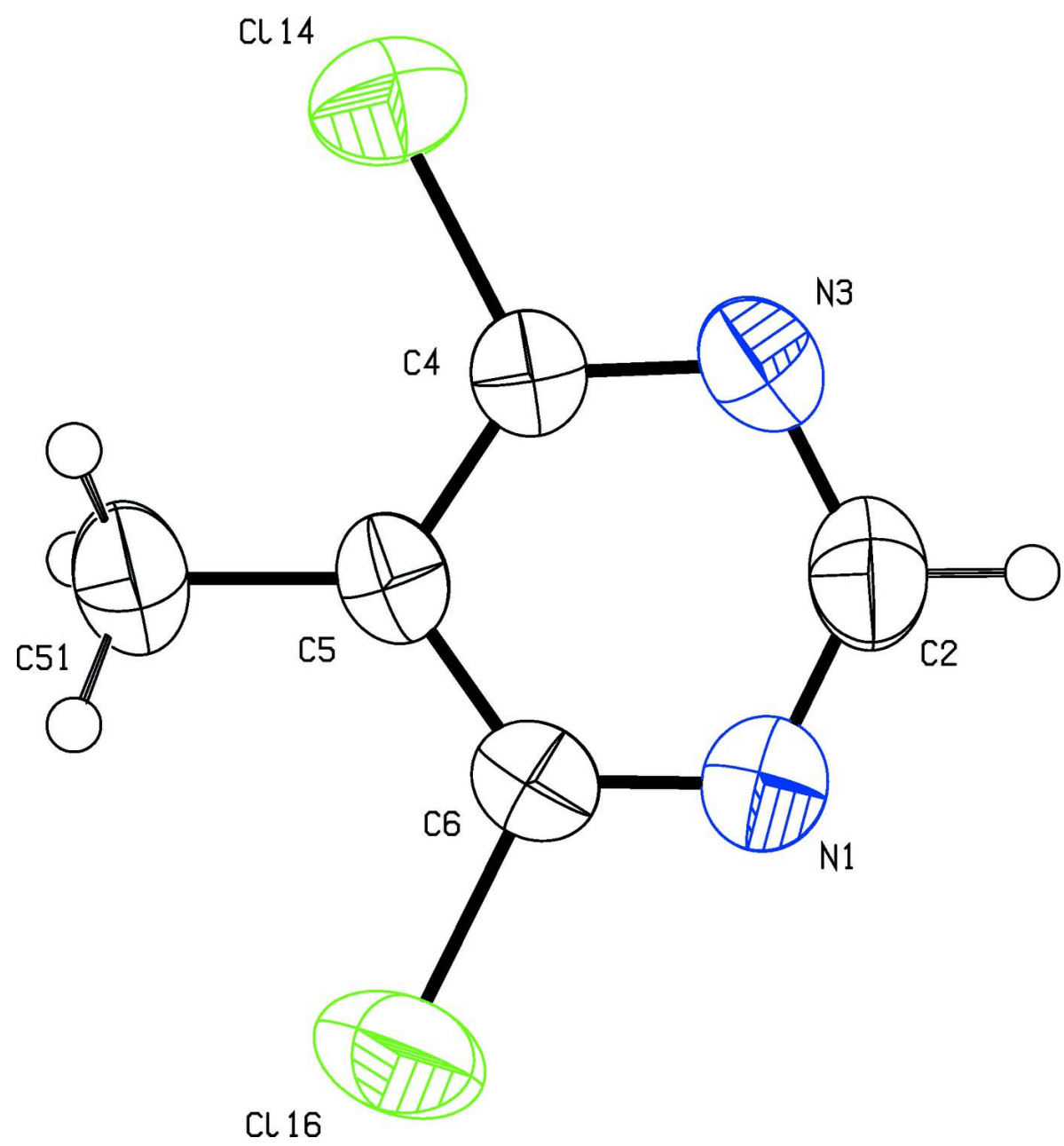

Figure 1

The molecular structure of the title compound, with atom labelling. Displacement ellipsoids drawn at the $50 \%$ probability level. 


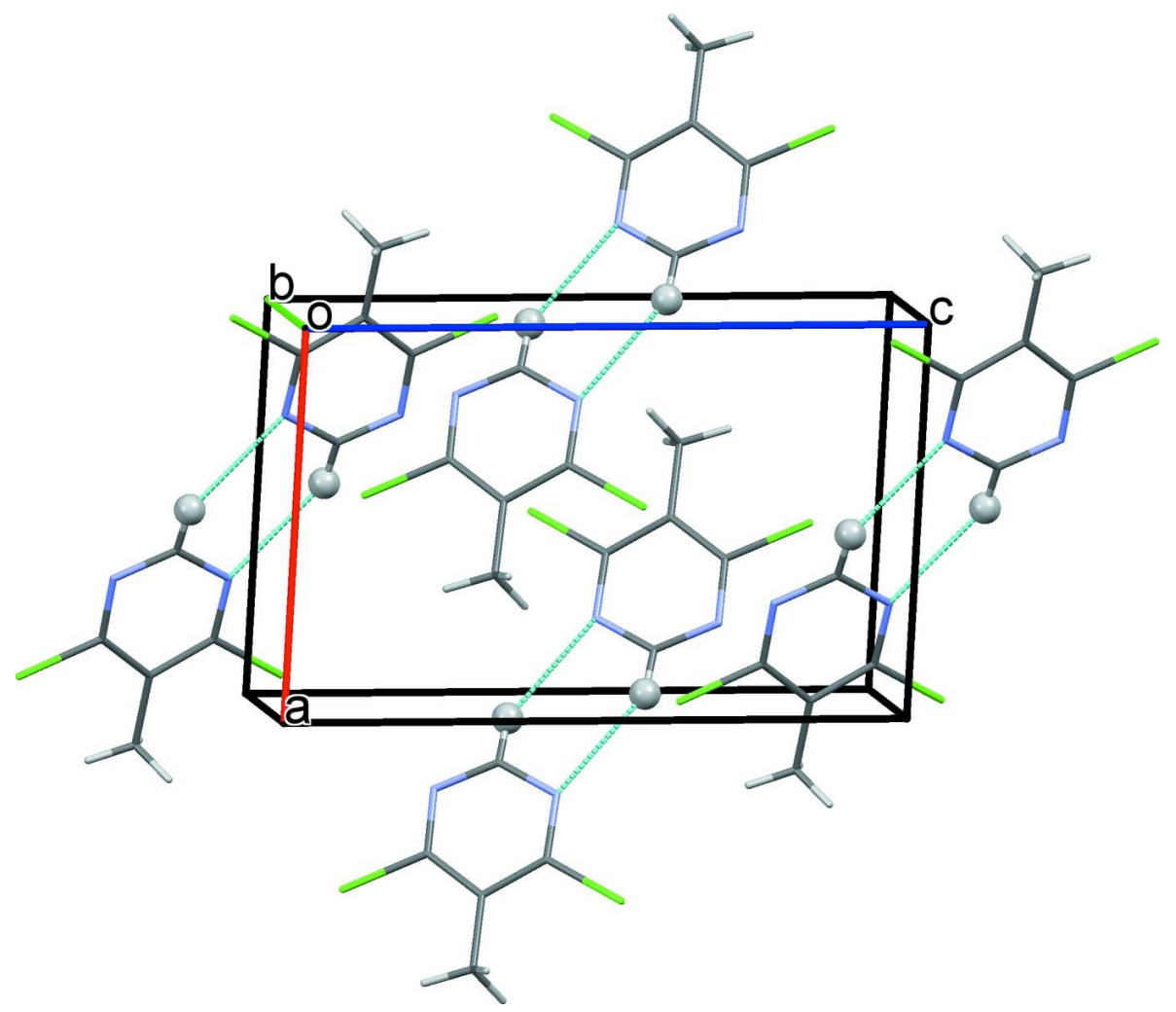

Figure 2

The crystal packing of the title compound, viewed along the $b$ axis. Hydrogen bonds are shown as dashed lines (see Table 1).

\section{4,6-Dichloro-5-methylpyrimidine}

\section{Crystal data}

\section{$\mathrm{C}_{5} \mathrm{H}_{4} \mathrm{Cl}_{2} \mathrm{~N}_{2}$}

$$
M_{r}=163.00
$$

Monoclinic, $P 2_{1} / n$

$a=7.463(5) \AA$

$b=7.827(5) \AA$

$c=11.790(5) \AA$

$\beta=93.233(5)^{\circ}$

$V=687.6(7) \AA^{3}$

$Z=4$

\section{Data collection}

Oxford Diffraction Xcalibur, Eos diffractometer

Radiation source: Enhance (Mo) X-ray Source Graphite monochromator

CCD rotation images, thin slices $\omega$ scans

Absorption correction: multi-scan

(CrysAlis PRO; Oxford Diffraction, 2013)

$T_{\min }=0.922, T_{\max }=0.934$
$F(000)=328$

$D_{\mathrm{x}}=1.575 \mathrm{Mg} \mathrm{m}^{-3}$

Mo $K \alpha$ radiation, $\lambda=0.71073 \AA$

Cell parameters from 776 reflections

$\theta=4.2-27.8^{\circ}$

$\mu=0.85 \mathrm{~mm}^{-1}$

$T=293 \mathrm{~K}$

Prism, colourless

$0.11 \times 0.10 \times 0.08 \mathrm{~mm}$

2347 measured reflections

1228 independent reflections

791 reflections with $I>2 \sigma(I)$

$R_{\text {int }}=0.099$

$\theta_{\text {max }}=25.2^{\circ}, \theta_{\min }=3.1^{\circ}$

$h=-8 \rightarrow 8$

$k=-9 \rightarrow 4$

$l=-14 \rightarrow 10$ 


\section{Refinement}

Refinement on $F^{2}$

Least-squares matrix: full

$R\left[F^{2}>2 \sigma\left(F^{2}\right)\right]=0.068$

$w R\left(F^{2}\right)=0.173$

$S=1.01$

1228 reflections

83 parameters

0 restraints

Primary atom site location: structure-invariant direct methods
Secondary atom site location: difference Fourier map

Hydrogen site location: inferred from neighbouring sites

$\mathrm{H}$-atom parameters constrained

$w=1 /\left[\sigma^{2}\left(F_{\mathrm{o}}^{2}\right)+(0.0729 P)^{2}\right]$ where $P=\left(F_{\mathrm{o}}^{2}+2 F_{\mathrm{c}}^{2}\right) / 3$

$(\Delta / \sigma)_{\max }<0.001$

$\Delta \rho_{\max }=0.39 \mathrm{e} \AA^{-3}$

$\Delta \rho_{\min }=-0.38$ e $\AA^{-3}$

Special details

Geometry. All esds (except the esd in the dihedral angle between two 1.s. planes) are estimated using the full covariance matrix. The cell esds are taken into account individually in the estimation of esds in distances, angles and torsion angles; correlations between esds in cell parameters are only used when they are defined by crystal symmetry. An approximate (isotropic) treatment of cell esds is used for estimating esds involving 1.s. planes.

Fractional atomic coordinates and isotropic or equivalent isotropic displacement parameters $\left(\AA^{2}\right)$

\begin{tabular}{lllll}
\hline & $x$ & $y$ & $z$ & $U_{\text {iso }} / U_{\text {eq }}$ \\
\hline C114 & $0.47393(16)$ & $0.21816(17)$ & $0.57181(8)$ & $0.0686(5)$ \\
C116 & $0.45631(17)$ & $0.4430(2)$ & $0.13763(9)$ & $0.0853(6)$ \\
N1 & $0.2066(5)$ & $0.4832(6)$ & $0.2775(3)$ & $0.0604(11)$ \\
N3 & $0.2153(5)$ & $0.3882(5)$ & $0.4695(3)$ & $0.0579(10)$ \\
C2 & $0.1383(6)$ & $0.4657(7)$ & $0.3784(4)$ & $0.0661(14)$ \\
H2 & 0.0251 & 0.5123 & 0.3865 & $0.079^{*}$ \\
C4 & $0.3744(5)$ & $0.3225(5)$ & $0.4544(3)$ & $0.0458(10)$ \\
C5 & $0.4659(5)$ & $0.3309(6)$ & $0.3546(3)$ & $0.0458(10)$ \\
C6 & $0.3650(6)$ & $0.4175(6)$ & $0.2685(3)$ & $0.0514(11)$ \\
C51 & $0.6464(6)$ & $0.2553(7)$ & $0.3426(4)$ & $0.0666(14)$ \\
H51A & 0.7041 & 0.3121 & 0.2823 & $0.100^{*}$ \\
H51B & 0.6344 & 0.1359 & 0.3252 & $0.100^{*}$ \\
H51C & 0.7175 & 0.2692 & 0.4124 & $0.100^{*}$ \\
\hline
\end{tabular}

Atomic displacement parameters $\left(\AA^{2}\right)$

\begin{tabular}{lllllll}
\hline & $U^{11}$ & $U^{22}$ & $U^{33}$ & $U^{12}$ & $U^{13}$ & $U^{23}$ \\
\hline C114 & $0.0846(9)$ & $0.0601(9)$ & $0.0604(6)$ & $-0.0009(7)$ & $-0.0019(6)$ & $0.0089(5)$ \\
C116 & $0.0934(11)$ & $0.1053(14)$ & $0.0592(7)$ & $-0.0109(9)$ & $0.0219(6)$ & $0.0116(6)$ \\
N1 & $0.057(2)$ & $0.061(3)$ & $0.063(2)$ & $0.005(2)$ & $0.0033(16)$ & $0.0012(19)$ \\
N3 & $0.057(2)$ & $0.057(3)$ & $0.0602(19)$ & $0.000(2)$ & $0.0160(15)$ & $-0.0040(17)$ \\
C2 & $0.050(2)$ & $0.074(4)$ & $0.075(3)$ & $0.008(3)$ & $0.005(2)$ & $-0.008(3)$ \\
C4 & $0.045(2)$ & $0.039(3)$ & $0.0537(19)$ & $-0.003(2)$ & $0.0041(16)$ & $-0.0034(18)$ \\
C5 & $0.042(2)$ & $0.039(3)$ & $0.057(2)$ & $-0.005(2)$ & $0.0089(16)$ & $-0.0056(17)$ \\
C6 & $0.054(2)$ & $0.053(3)$ & $0.0475(18)$ & $-0.004(2)$ & $0.0070(16)$ & $-0.0023(18)$ \\
C51 & $0.051(3)$ & $0.070(4)$ & $0.081(3)$ & $0.007(3)$ & $0.016(2)$ & $-0.002(2)$ \\
\hline
\end{tabular}


Geometric parameters $\left(\AA,{ }^{o}\right)$

\begin{tabular}{|c|c|c|c|}
\hline $\mathrm{C} 114-\mathrm{C} 4$ & $1.737(4)$ & $\mathrm{C} 4-\mathrm{C} 5$ & $1.395(5)$ \\
\hline $\mathrm{C} 116-\mathrm{C} 6$ & $1.733(4)$ & $\mathrm{C} 5-\mathrm{C} 6$ & $1.403(6)$ \\
\hline $\mathrm{N} 1-\mathrm{C} 6$ & $1.299(6)$ & $\mathrm{C} 5-\mathrm{C} 51$ & $1.486(6)$ \\
\hline $\mathrm{N} 1-\mathrm{C} 2$ & $1.327(5)$ & $\mathrm{C} 51-\mathrm{H} 51 \mathrm{~A}$ & 0.9600 \\
\hline $\mathrm{N} 3-\mathrm{C} 4$ & $1.315(5)$ & $\mathrm{C} 51-\mathrm{H} 51 \mathrm{~B}$ & 0.9600 \\
\hline $\mathrm{N} 3-\mathrm{C} 2$ & $1.335(5)$ & $\mathrm{C} 51-\mathrm{H} 51 \mathrm{C}$ & 0.9600 \\
\hline $\mathrm{C} 2-\mathrm{H} 2$ & 0.9300 & & \\
\hline $\mathrm{C} 6-\mathrm{N} 1-\mathrm{C} 2$ & $115.4(3)$ & $\mathrm{C} 6-\mathrm{C} 5-\mathrm{C} 51$ & $125.2(4)$ \\
\hline $\mathrm{C} 4-\mathrm{N} 3-\mathrm{C} 2$ & $114.8(3)$ & $\mathrm{N} 1-\mathrm{C} 6-\mathrm{C} 5$ & $125.9(3)$ \\
\hline $\mathrm{N} 1-\mathrm{C} 2-\mathrm{N} 3$ & $126.8(4)$ & $\mathrm{N} 1-\mathrm{C} 6-\mathrm{Cl16}$ & $115.6(3)$ \\
\hline $\mathrm{N} 1-\mathrm{C} 2-\mathrm{H} 2$ & 116.6 & $\mathrm{C} 5-\mathrm{C} 6-\mathrm{Cl} 16$ & $118.5(3)$ \\
\hline $\mathrm{N} 3-\mathrm{C} 2-\mathrm{H} 2$ & 116.6 & $\mathrm{C} 5-\mathrm{C} 51-\mathrm{H} 51 \mathrm{~A}$ & 109.5 \\
\hline $\mathrm{N} 3-\mathrm{C} 4-\mathrm{C} 5$ & $125.7(4)$ & $\mathrm{C} 5-\mathrm{C} 51-\mathrm{H} 51 \mathrm{~B}$ & 109.5 \\
\hline $\mathrm{N} 3-\mathrm{C} 4-\mathrm{Cl14}$ & $115.1(3)$ & $\mathrm{H} 51 \mathrm{~A}-\mathrm{C} 51-\mathrm{H} 51 \mathrm{~B}$ & 109.5 \\
\hline $\mathrm{C} 5-\mathrm{C} 4-\mathrm{Cl14}$ & $119.2(3)$ & $\mathrm{C} 5-\mathrm{C} 51-\mathrm{H} 51 \mathrm{C}$ & 109.5 \\
\hline $\mathrm{C} 4-\mathrm{C} 5-\mathrm{C} 6$ & $111.4(4)$ & $\mathrm{H} 51 \mathrm{~A}-\mathrm{C} 51-\mathrm{H} 51 \mathrm{C}$ & 109.5 \\
\hline $\mathrm{C} 4-\mathrm{C} 5-\mathrm{C} 51$ & $123.4(4)$ & $\mathrm{H} 51 \mathrm{~B}-\mathrm{C} 51-\mathrm{H} 51 \mathrm{C}$ & 109.5 \\
\hline $\mathrm{C} 6-\mathrm{N} 1-\mathrm{C} 2-\mathrm{N} 3$ & $-0.2(8)$ & $\mathrm{C} 114-\mathrm{C} 4-\mathrm{C} 5-\mathrm{C} 51$ & $-0.2(6)$ \\
\hline $\mathrm{C} 4-\mathrm{N} 3-\mathrm{C} 2-\mathrm{N} 1$ & $-0.7(8)$ & $\mathrm{C} 2-\mathrm{N} 1-\mathrm{C} 6-\mathrm{C} 5$ & $0.5(8)$ \\
\hline $\mathrm{C} 2-\mathrm{N} 3-\mathrm{C} 4-\mathrm{C} 5$ & $1.3(7)$ & $\mathrm{C} 2-\mathrm{N} 1-\mathrm{C} 6-\mathrm{C} 116$ & $-179.0(4)$ \\
\hline $\mathrm{C} 2-\mathrm{N} 3-\mathrm{C} 4-\mathrm{Cl} 14$ & $-178.9(4)$ & $\mathrm{C} 4-\mathrm{C} 5-\mathrm{C} 6-\mathrm{N} 1$ & $0.1(7)$ \\
\hline $\mathrm{N} 3-\mathrm{C} 4-\mathrm{C} 5-\mathrm{C} 6$ & $-1.1(6)$ & $\mathrm{C} 51-\mathrm{C} 5-\mathrm{C} 6-\mathrm{N} 1$ & $179.5(5)$ \\
\hline $\mathrm{Cl} 14-\mathrm{C} 4-\mathrm{C} 5-\mathrm{C} 6$ & $179.2(3)$ & $\mathrm{C} 4-\mathrm{C} 5-\mathrm{C} 6-\mathrm{Cl1} 6$ & $179.6(3)$ \\
\hline $\mathrm{N} 3-\mathrm{C} 4-\mathrm{C} 5-\mathrm{C} 51$ & $179.5(4)$ & $\mathrm{C} 51-\mathrm{C} 5-\mathrm{C} 6-\mathrm{C} 116$ & $-1.0(7)$ \\
\hline
\end{tabular}

Hydrogen-bond geometry $\left(\AA,{ }^{\circ}\right)$

\begin{tabular}{lllll}
\hline$D-\mathrm{H} \cdots A$ & $D-\mathrm{H}$ & $\mathrm{H} \cdots A$ & $D \cdots A$ & $D-\mathrm{H} \cdots A$ \\
\hline $\mathrm{C} 2-\mathrm{H} 2 \cdots \mathrm{N} 3^{\mathrm{i}}$ & 0.93 & 2.66 & $3.468(6)$ & 146 \\
\hline
\end{tabular}

Symmetry code: (i) $-x,-y+1,-z+1$. 